\title{
Controlled Particle Size and Synthesizing Mechanism of Microsphere of Poly(MMA-BuMA) Prepared by Emulsion Polymerization
}

\author{
By Yuguang FENG, Shihua HUANG, ${ }^{*}$ and Feng TENG
}

Based on the characteristics of potassium peroxydisulfate (KPS) and sodium dodecyl sulfate (SDS), a series of microspheres of poly(methyl methacrylate-co-butyl methacrylate) [poly(MMA-BuMA)] were synthesized by emulsion polymerization. The particle size of poly(MMA-BuMA) microspheres increased from 65 to $1144 \mathrm{~nm}$ with varing amounts of KPS and SDS as well; and the mean square errors increased from 5 to 6253 with various amounts of KPS and SDS as well. It was found that the analyzed mechanism of emulsifying polymerization can be carried out under non-equilibrium state, and the analyzed mechanism of emulsifier-free polymerization and the oligomer-micellar nucleation theory were coming into conflict.

KEY WORDS: Emulsion Polymerization / Poly(MMA-BuMA) / Particle Size / Mechanisms /

As known well, emulsion polymerization has two different reacting styles, emulsifying polymerization and emulsifier-free polymerization, which have their own mechanisms.

To elucidate emulsifier-free polymerization, both of homogeneous nucleation theory and oligomer-micellar nucleation theory had been induced. ${ }^{1-11}$ That polymer microsphere is grown with coiled polymer chains in homogeneous phase is the true meaning of homogeneous nucleation theory. The foundation of oligomer-micellar nucleation theory is that an oligomer surfactant can be synthesized with corresponding monomers and initiator in the initial stage.

With regard to emulsifying polymerization, the fact that the diameter of the obtained polymer particles decreased drastically as surfactant concentration increased over critical micellar concentration (CMC) of the surfactant and decreased continuously as surfactant concentration increased can be predicted by the former reported fact ${ }^{12,13}$ that the number of polymer particles increases drastically as surfactant concentration increases over CMC and increases continuously as surfactant concentration increases. It is essential that HLB value of monomer mixture must be close to another value of surfactants used. ${ }^{14}$ Thus, the equilibrium of dispersed liquid or resultant latex can be preserved in order to avoid gelatin. Moreover, various particle sizes of microspheres are dominated by physical methods such as agitating speed, dispersing time, etc.

In this article, stable poly(MMA-BuMA) latex was prepared separately by emulsifying polymerization and emulsifier-free polymerization. In emulsifying polymerization, the latex was synthesized under a wide gap of HLB value between monomers and emulsifying agents, and the particle sizes of poly(MMA-BuMA) microspheres were controlled with various amounts of KPS and SDS. This will be a supplementary to mechanism of emulsifying polymerization. The emulsifier-free polymerized latex was obtained against oligomer-micellar nucleation theory. The characteristics of used polymerizations are that the initial dispersing states are non-equilibrium states, and the resultant latexes are stable. This will be replenishment to mechanism of emulsifier-free polymerization.

\section{EXPERIMENTAL}

\section{Materials}

Both methyl methacrylate (MMA) and butyl methacrylate (BuMA) were purchased from Alfa Aesar Chemical Co., USA, and purified using cuprous hydrochloride and stored at $-5{ }^{\circ} \mathrm{C}$ prior to use. Potassium peroxydisulfate (KPS) was used as an initiator without further purification. Sodium dodecyl sulfate (SDS) was a surfactant. Deionized water, whose resistance is more than $10 \mathrm{M} \Omega$, was prepared by our laboratory.

\section{Polymerization}

The emulsifier-free polymerization ingredients consist of medium, which is deionized water, co-monomer mixture, and various amounts of KPS. No stabilizer was employed. The total amount of monomers was set at $45 \mathrm{wt} \%$ for the medium and $0.37-2.2 \mathrm{wt} \%$ of KPS with respect to the total amount of the monomers used. After charging KPS and deionized water in a $250 \mathrm{~mL}$ flask with stir, condenser, thermometer and gas pipe, the agitation began to run until KPS was dissolved into deionized water. Then co-monomer mixture was poured into aqueous solution of KPS with an agitation speed of $300 \mathrm{rpm}$, and argon was purged until the end of reaction. After comonomer mixture was dispersed for $3 \mathrm{~h}$, emulsifier-free polymerization was carried out in a water bath at $70^{\circ} \mathrm{C}$ for $5 \mathrm{~h}$, then at $80^{\circ} \mathrm{C}$ for $1 \mathrm{~h}$. After the completion of polymerization, the resultant latexes were obtained by filter.

Key Laboratory of Luminescence and Optical Information, Ministry of Education, Institute of Optoelectronic Technology, Beijing Jiaotong University, 3 
Table I. The Reaction Recipe of Microsphere of Poly(MMA-BuMA)

\begin{tabular}{|c|c|c|c|c|c|c|c|c|c|c|c|c|}
\hline \multirow{2}{*}{$\begin{array}{l}\text { Medium, } \\
\text { water/g }\end{array}$} & \multicolumn{2}{|c|}{ Monomer 45 wt \% (to medium) } & \multirow{2}{*}{\multicolumn{2}{|c|}{$\mathrm{KPS} / \mathrm{g}_{\mathrm{SDS} / \mathrm{g}}$}} & \multicolumn{8}{|c|}{ SDS, $0.0-2.0 \mathrm{wt} \%$ (to water) } \\
\hline & $\mathrm{MMA} / \mathrm{g}$ & BuMA/g & & & 0.0 & 0.1 & 0.2 & 0.4 & 0.6 & 0.8 & 1.0 & 1.2 \\
\hline \multirow[t]{12}{*}{60} & \multirow[t]{12}{*}{13.5} & \multirow[t]{12}{*}{13.5} & \multirow{5}{*}{ 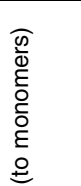 } & 0.1 & 591 & 103 & 82 & 64 & 62 & 60 & 66 & 73 \\
\hline & & & & 0.2 & 675 & 109 & 86 & 76 & 68 & 58 & 56 & 49 \\
\hline & & & & 0.3 & 803 & 121 & 75 & 86 & 73 & 58 & 52 & 50 \\
\hline & & & & 0.4 & 775 & 117 & 67 & 94 & 74 & 49 & 60 & 66 \\
\hline & & & & 0.5 & 1032 & 185 & 136 & 124 & 74 & 56 & 53 & 51 \\
\hline & & & \multirow{3}{*}{ 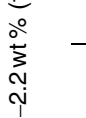 } & 0.6 & 1144 & 245 & 201 & 135 & 76 & 65 & 63 & 67 \\
\hline & & & & 0.1 & 601 & 188 & 29 & 25 & 39 & 53 & 49 & 44 \\
\hline & & & & 0.2 & 1801 & 72 & 24 & 37 & 33 & 30 & 36 & 42 \\
\hline & & & n़ & 0.3 & 2470 & 125 & 53 & 28 & 38 & 49 & 27 & 5 \\
\hline & & & \multirow{3}{*}{$\begin{array}{l}0 \\
\text { की } \\
\frac{0}{y}\end{array}$} & 0.4 & 3149 & 76 & 73 & 21 & 25 & 29 & 26 & 23 \\
\hline & & & & 0.5 & 12129 & 82 & 35 & 89 & 43 & 13 & 18 & 6 \\
\hline & & & & 0.6 & 6253 & 102 & 93 & 68 & 55 & 20 & 35 & 54 \\
\hline
\end{tabular}

*Data in the above black frame belong to particle size. Data in the below black frame belong to mean square error.

The emulsifying polymerization ingredients consist of medium, which is deionized water, co-monomer mixture, and various amounts of KPS and SDS. The total amount of monomers was set at $45 \mathrm{wt} \%$ for the medium, and the amount of SDS was set from $0.16 \mathrm{wt} \%$ to $2.0 \mathrm{wt} \%$, and $0.37-2.2 \mathrm{wt} \%$ of KPS with respect to the total amount of the monomers used. After charging KPS, SDS and deionized water in a $250 \mathrm{~mL}$ flask with stir, condenser, thermometer and gas pipe, the agitation began to run until KPS and SDS were dissolved into deionized water. Then co-monomer mixture was poured into aqueous solution of KPS and SDS with an agitation speed of $300 \mathrm{rpm}$, and argon was purged until the end of reaction. After co-monomer mixture was dispersed for $3 \mathrm{~h}$, emulsifier-free polymerization was carried out in a water bath at $70^{\circ} \mathrm{C}$ for $5 \mathrm{~h}$, then at $80^{\circ} \mathrm{C}$ for $1 \mathrm{~h}$. After the completion of polymerization, the resultant latexes were obtained by filter. Both two reaction recipes are listed in Table I.

\section{Characterization}

A Hitachi TEM (Transmission Electron Microscopy) H-700 was used to study the morphology of poly(MMA-BuMA) particles. Number-average diameter $\left(D_{n}\right)$ and the mean square error were achieved using Scion image analyzer software by counting individual 200 particles from TEM microphotographs.

Differential scanning calorimeter (DSC, NETZSCH, 200PC, Germany) was used to observe glass transition temperature of poly(MMA-BuMA) particles. The samples were heated up to $80^{\circ} \mathrm{C}$ with a heating rate of $10^{\circ} \mathrm{C} / \mathrm{min}$ under a nitrogen atmosphere, and were kept warm at $80^{\circ} \mathrm{C}$ for $10 \mathrm{~min}$. Then, the samples were quench-cooled at a maximum cooling rate with liquid nitrogen before reheated in a second time. The glass transition temperature was collected in the second heating.

FT-IR (FT-IR-8400, Shimadzu, Japan) was performed on a spectrometer. All the samples were mixed with spectroscopic grade potassium bromide before they were placed in the sample cell, and diffuse reflectance spectra were scanned over a range of $500-4000 \mathrm{~cm}^{-1}$.
An X-ray diffraction instrument (DX-D1, Shimadzu Corporation, Japan) was used to analyze the surface and permutation of microspheres with $\mathrm{Cu} \mathrm{K} \alpha$ radiation $(35 \mathrm{kV}, 20 \mathrm{~mA})$ scanning over a range of $0-90^{\circ}\left(0^{\circ}<2 \theta<90^{\circ}\right)$.

The mean square error of particle size was calculated using an equation as follows:

$$
\sigma=\frac{1}{n} \sum_{i=1}^{n}\left(X_{i}-\bar{X}\right)^{2}
$$

$\sigma:$ mean square error

$\bar{X}$ : Value of number-average diameter of $n$ microspheres $X_{i}$ : Value of diameter of the $i$-th particle

\section{RESULTS}

Figure 1 exhibits the DSC thermogram of the poly(MMABuMA) particles prepared in deionized water.

The glass transition temperature of poly(MMA-BuMA) was obtained at $52.5^{\circ} \mathrm{C}$ as seen in Figure 1. Moreover, the designed glass transition temperature of poly(MMA-BuMA) can be calculated using an empirical equation ${ }^{15}$ as follows:

$$
\frac{1}{T_{\mathrm{g}}}=\frac{W_{1}}{T_{\mathrm{g} 1}}+\frac{W_{2}}{T_{\mathrm{g} 2}}
$$

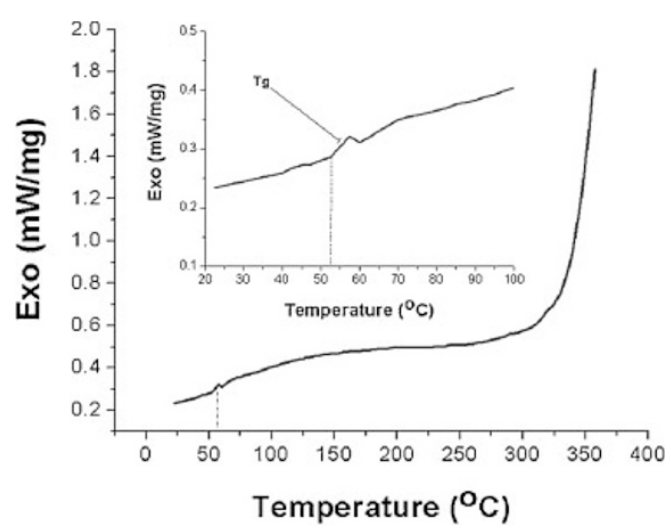

Figure 1. DSC thermogram of poly(MMA-BuMA) microspheres. 


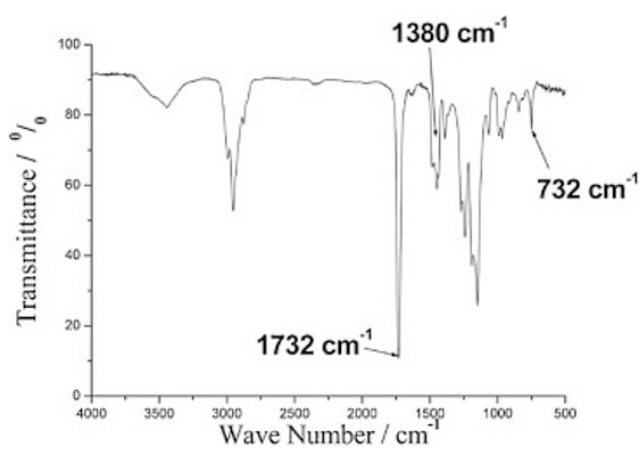

Figure 2. FT-IR spectrum of poly(MMA-BuMA) prepared by emulsion polymerization.

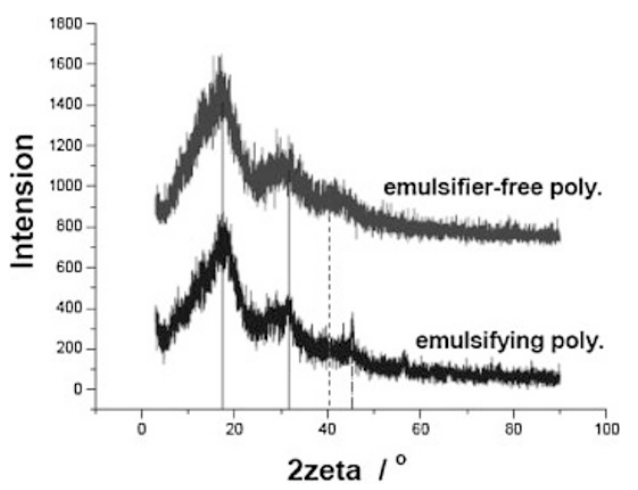

Figure 3. XRD of poly(MMA-BuMA) microspheres.

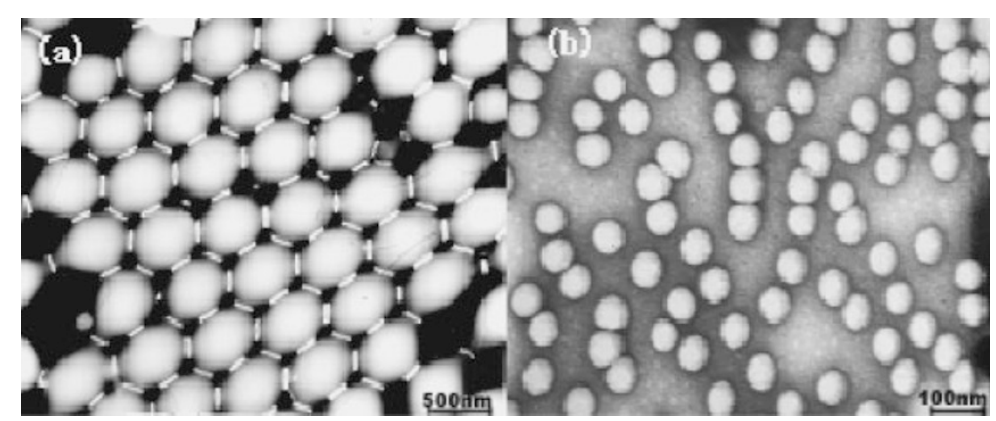

Figure 4. TEM photographs of poly(MMA-BuMA) prepared by (a) emulsifier-free and (b) emulsifying polymerizations.

$T_{\mathrm{g}}$ : The glass transition temperature of resultant polymer, $\mathrm{K}$;

$T_{\mathrm{g} 1}$ : The glass transition temperature of homopolymer polymerized with monomer $1, \mathrm{~K}$;

$T_{\mathrm{g} 2}$ : The glass transition temperature of homopolymer polymerized with monomer $2, \mathrm{~K}$;

$W_{1}$ : The mass fraction of monomer 1 with respect to the mass fraction of monomer 2;

$W_{2}$ : The mass fraction of monomer 2 with respect to the mass fraction of monomer 1.

According to Table I, both mass fractions of MMA and BuMA are 0.5; The glass transition temperature of pure poly(MMA) was verified at $95+273.15 \mathrm{~K} ;{ }^{16}$ and the glass transition temperature of pure poly(BuMA) was verified at $20+273.15 \mathrm{~K} \cdot{ }^{17}$ Thus, the glass transition temperature of poly(MMA-BuMA) was calculated at $53{ }^{\circ} \mathrm{C}$ approaching to $52.5^{\circ} \mathrm{C}$. So it was characterized that the microsphere of poly(MMA-BuMA) was prepared with mass-equal monomers of MMA and BuMA.

In order to confirm the reaction between MMA and BuMA, the FT-IR spectrum was studied and is shown in Figure 2. The FT-IR spectrum had the strongest peak at $1732 \mathrm{~cm}^{-1}$ corresponding to $\mathrm{C}=\mathrm{O}$ stretching adsorption bond of ester groups, and the characteristic peaks $1380 \mathrm{~cm}^{-1}$ and $732 \mathrm{~cm}^{-1}$ corresponding to $-\mathrm{CH}_{3}$ and $-\mathrm{C}_{4} \mathrm{H}_{9}$ stretching adsorption bond. The $-\mathrm{CH}_{2}-$ stretching of the methacrylate group is at 2800$3000 \mathrm{~cm}^{-1}$.

As far as the special characterization of poly(MMA-BuMA) prepared by emulsifier-free polymerization, Figure 3 exhibits the X-ray diffraction graph to analyze the sulfate group around the microspheres and permutation between microspheres.

The XRD chat had strong peaks at $17.53^{\circ}$ and $31.55^{\circ}$ corresponding to permutation between microspheres and weaker peak at $40.28^{\circ}$ corresponding to sulfate groups and another weaker peak at $45.19^{\circ}$ corresponding to residual sodium hydrochloride.

Based on the content mentioned above, TEM photographs of poly(MMA-BuMA) microspheres prepared by emulsifying polymerization and emulsifier-free polymerization are shown in Figure 4.

\section{DISCUSSION}

According to the data from Table I, the three-dimensional representation of particle size and amounts of KPS and SDS was shown in Figure 5.

As seen in Figure 5(a), the highest polygonal line of particle size belongs to poly(MMA-BuMA) microspheres prepared without any amount of SDS. That is to say, the particle size of poly(MMA-BuMA) microspheres prepared by emulsifier-free polymerization was larger than emulsifying polymerization. Meanwhile, the particle size of poly(MMA-BuMA) microspheres prepared by emulsifier-free polymerization dramatically increased from $591 \mathrm{~nm}$ to $1144 \mathrm{~nm}$ with increasing amount of KPS from $0.1 \mathrm{~g}$ to $0.6 \mathrm{~g}$. And the particle size of poly(MMA-BuMA) microspheres prepared with various amounts of KPS and SDS was shown in Figure 5(b). 

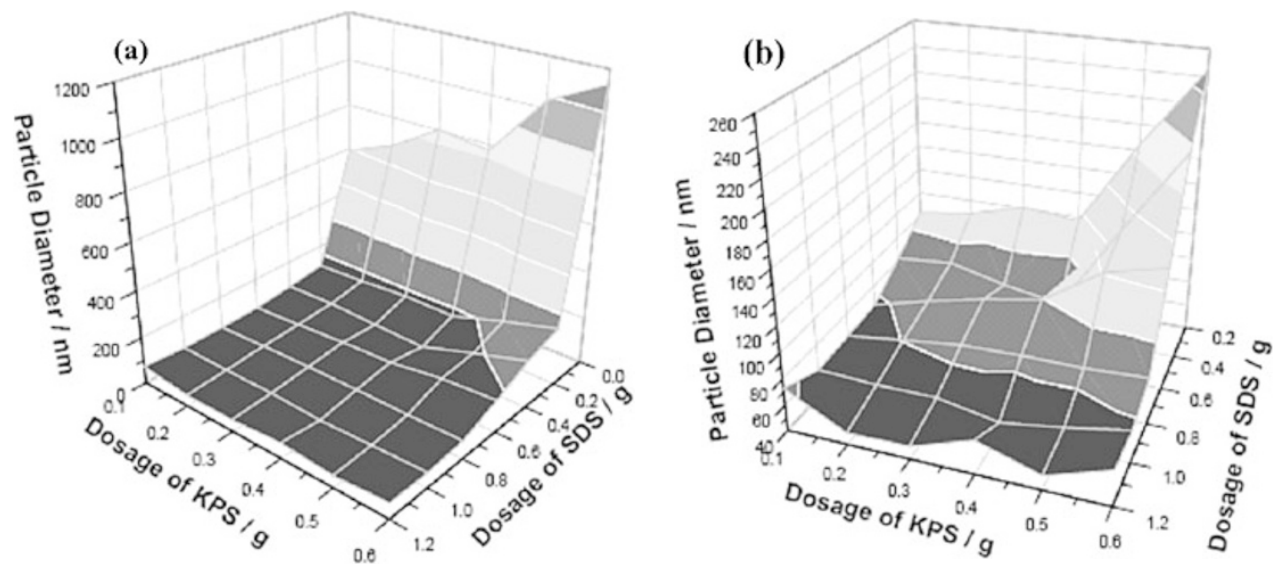

Figure 5. The particle size of poly(MMA-BuMA) microspheres upon the amounts of KPS and SDS.
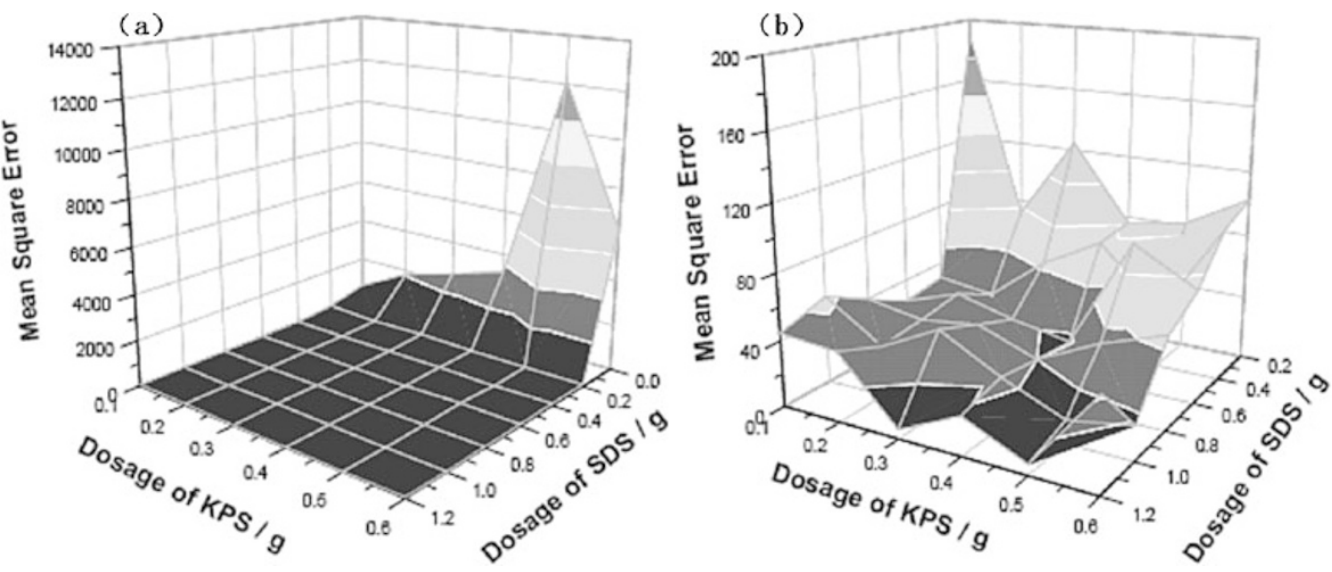

Figure 6. Mean square error of poly(MMA-BuMA) particle size upon the amounts of KPS and SDS.

The topography of poly(MMA-BuMA) microsphere diameters upon various amounts of KPS and SDS appears a quarter of cone, which has a tiptop at (KPS $0.6 \mathrm{~g}$, SDS $0.2 \mathrm{~g}$, diameter $201 \mathrm{~nm}$ ). As the amount of SDS was set at $0.2 \mathrm{~g}$, particle size of poly(MMA-BuMA) microspheres increased from $82 \mathrm{~nm}$ to $201 \mathrm{~nm}$ with increasing amounts of KPS from $0.1 \mathrm{~g}$ to $0.6 \mathrm{~g}$; and as the amount of KPS was set at $0.6 \mathrm{~g}$, particle size of poly(MMA-BuMA) microspheres increased from $67 \mathrm{~nm}$ to $201 \mathrm{~nm}$ with decreasing amounts of SDS from $1.2 \mathrm{~g}$ to $0.2 \mathrm{~g}$. Meanwhile, the side of the cone also gradually went up to the tiptop with increasing amounts of KPS and decreasing amounts of SDS. Thus, KPS initiating radicals has other effects upon the stability of latex and the particle size of microspheres; and SDS prevents the flocculation of growing particles even if stabledispersing spherical polymer particles were obtained by electrostatic stabilization in the presence of SDS.

Figure 6 represents mean square errors of poly(MMABuMA) particle size upon various amounts of KPS and SDS. As seen in Figure 6(a), the highest polygonal line belongs to mean square errors of poly(MMA-BuMA) microspheres prepared by emulsifier-free polymerization (The amount of SDS was set at 0), and mean square errors increased from 601 to 12129 with increasing amounts of KPS from $0.1 \mathrm{~g}$ to $0.6 \mathrm{~g}$ due to mechanical mixture in the initial dispersing stage. The turbid liquid composed of deionized water and monomers was out of non-equilibrium state without electrostatic stabilizer, and then the highest polygonal line existed. On the other hand, as seen in Figure 6(b), mean square errors decreased wavily with increasing amounts of SDS from $0.2 \mathrm{~g}$ to $1.2 \mathrm{~g}$. Although HLB value of SDS is far to HLB values of monomers, micelles of $\mathrm{O} / \mathrm{W}$ style can be controlled with SDS, which is one of electrostatic stabilizers and works in coordination with stir. And then the dispersion between deionized water and monomers increased better with increasing amounts of SDS until the particle sizes of resultant microspheres decreased and the corresponding uniformity was improved. As far as KPS be concerned, the amounts of KPS operated less on the mean square errors while the amount of SDS was set at a constant.

In general, emulsifying polymerization is carried out with the approximation of HLB value between monomers and electrostatic stabilizer used. In this paper, poly(MMA-BuMA) microsphere was prepared with a gap of HLB value between SDS and monomers in dispersing stage. Thus, the turbid liquid without the approximation of HLB value between SDS and monomers was in such a non-equilibrium state that precipitation occurred as soon as stirring was stopped. The turbid 


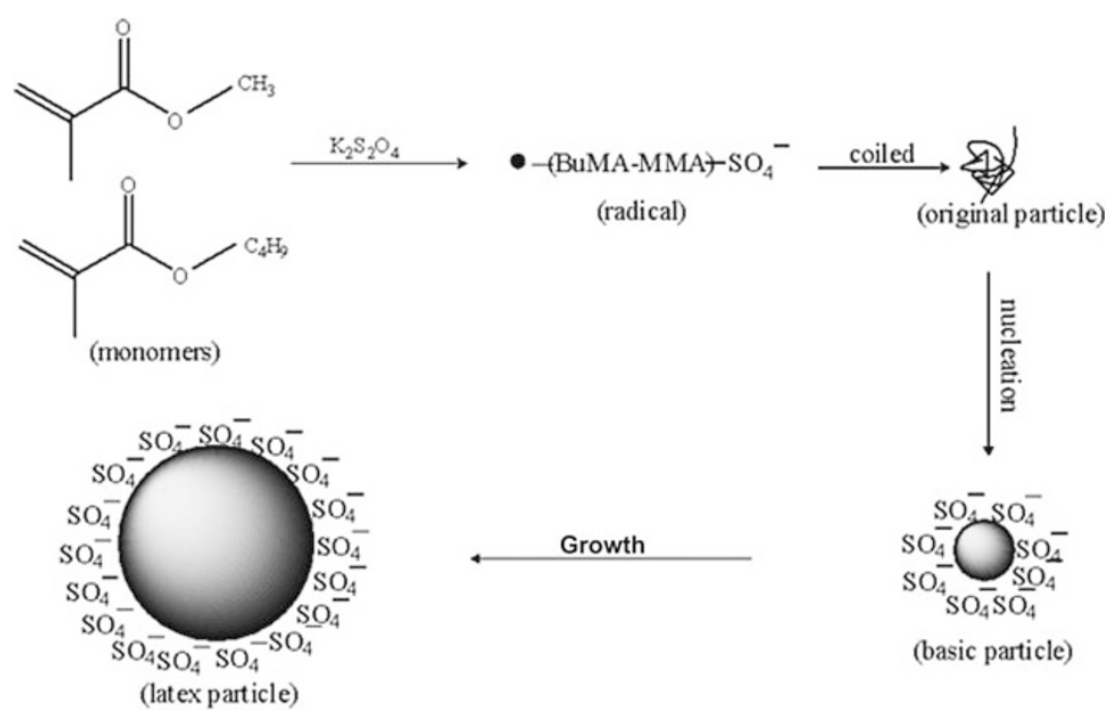

Figure 7. The process of emulsifier-free polymerization.

liquid was still changed into stable latex with KPS initiating radical and linking polymer chains. And then the ends of resultant polymer chains were combined with sulfate groups, which are hydrophilic groups. So it was confirmed that KPS operated on the latex stability and particle size, SDS operated on particle size and the mean square error, and emulsifying polymerization in this article can be carried out by the intermediate mechanism of emulsifying polymerization and emulsifier-free polymerization.

As far as emulsifier-free polymerization is concerned, the oligomer-micellar nucleation theory seems to expound this polymerization well. The theory's quintessence is that the oligomer surfactant is prepared and keeps the micelles of $\mathrm{O} / \mathrm{W}$ style stable with sulfate groups combined at the end of the oligomer in the initial reaction stage. Thus, the emulsifier-free polymerization has been changed into emulsifying polymerization with oligomer surfactant, and then the particle sizes and mean square errors corresponding resultant microspheres should decrease with increasing amounts of KPS, which is one of raw materials preparing the oligomer surfactant. On the contrary, the oligomer-micellar nucleation theory does not conform to the reality mentioned above. The phenomenon was analyzed as follows:

a. The polymer chain grows so instantly in the initial radical polymerization that corresponding oligomer is prepared more difficultly except polymer.

b. If the HLB value of oligomer surfactant was so close to the HLB value of monomer mixture that the particle size of resultant polymer would have decreased with increasing amounts of oligomer surfactant prepared with KPS which is at the end of the oligomer chain; that is to say, the particle size of resultant polymer would have decreased with increasing amounts of KPS. Actually, the theory does not conform to the reality mentioned above.

Thus, the only available mechanism of emulsifier-free polymerization was suggested to be shown as Figure 7.
Similarly, the fact that at low surfactant concentration the diameter of the obtained polymer particles increased as KPS concentration increases was reported in the published paper dealing the emulsifier-free styrene polymerization initiated by KPS, ${ }^{18}$ which is clearly explained by the electric screening effect of salts (KPS is a kind of salt) which make the generated precursor particle unstable against coagulation. In this paper, emulsifier-free polymerization is a pure reaction without any surfactant, and methacrylate monomers were used into it. Then works in this paper may be a supplementary for the relevant theory, and further more experiments will be done to improve and perfect the quintessence of emulsion polymerization.

\section{CONCLUSIONS}

Poly(MMA-BuMA) microsphere was prepared separately by emulsifier-free polymerization and emulsifying polymerization and stable latex was obtained. The dispersed turbid liquid of deionized water and monomers was in the nonequilibrium state before emulsifying polymerization which was initiated with KPS. The particle sizes and mean square errors of poly(MMA-BuMA) microspheres being analyzed, the oligomer-micellar nucleation theory can not conform the reality in this paper. The intensive study of the evidence for the mechanism of emulsion polymerization will be disclosed shortly.

Received: September 9, 2008

Accepted: December 17, 2008

Published: February 4, 2009

\section{REFERENCES}

1. R. M. Fitch, Br. Polym. J., 5, 467 (1973).

2. J. W. Goodwin and C. C. Ho, Br. Polym. J., 5, 347 (1973).

3. F. K. Hansen and J. Ugelstad, J. Polym. Sci., Polym. Chem. Ed., 16, 1953 (1978). 
4. P. J. Feeney, D. H. Napper, and R. G. Gilbert, Macromolecules, 20, 2922 (1987).

5. H. J. Van den Hui and J. W. Vanderhoff, Collid Interface Sci., 28, 336 (1986).

6. J. W. Vanderhoff, J. Polym. Sci. Polym. Symp., 23, 161 (1985).

7. A. R. Goodall, M. C. Wilkinson and J. Hearn, J. Polym. Sci., Part A: Polym. Chem., 15, 2193 (1977).

8. Z. Song, J. Colloid Interface Sci., 128, 486 (1989).

9. Z. Song, J. Colloid Interface Sci., 128, 501 (1989).

10. J. Brandrup and E. H. Immergut, "Polymer Handbook," WileyInterscience, New York, 1975.

11. A. R. Goodall and M. C. Wilkinson, "Polymer Colloids II," R. M. Fitch, Ed., New York, Plenum, 1980.
12. M. Nomura, M. Harada, W. Eguchi, and S. Nagata, J. Appl. Polym. Sci., 16, 835 (1972).

13. M. Nomura, A. Yamada, S. Fujita, A. Sugimoto, J. Ikoma, and K. Fujita, J. Polym. Sci., Part A: Polym. Chem., 29, 987 (1991).

14. I. M. Kolthoff and I. K. Miller, J. Am. Chem. Soc., 73, 3055 (1951).

15. T. G. Fox, Bull. Am. Phys. Soc., 1, 123 (1956).

16. H. Ohmori, K. Arimitsu, K. Kudo, Y. Hayashi, and K. Ichimura, J. Photopolym. Sci. Technol., 9, 25 (1996).

17. J. Hrouz and J. Janácek, J. Macromol. Sci. Part B Phys., 5, 245 (1971).

18. A. Kotera, K. Furusawa, and Y. Takeda, Kolloid Z. Z. Polym., 239, 677 (1970). 